\title{
Informe preliminar: Revisión de certificados de defunción por SIDA no notificados. Chile, 1997-2003
}

\author{
COMISIÓN NACIONAL DE SIDA-CONASIDA \\ MINISTERIO DE SALUD DE CHILE
}

\section{Preliminary report: Review of AIDS death certificates not reported. Chile, 1997-2003}

Key words: SIDA, Muerte, Certificado de defunción.

Palabras claves: AIDS, Death, Death certificate.

Toda defunción debe inscribirse en el Servicio de Registro Civil e Identificación de la comuna donde ocurrió la muerte, dentro del plazo de tres días a contar de la fecha de fallecimiento. Este plazo vence a las 24 horas del día en que se cumplen las 72 horas desde el momento de la defunción (Ley 4.808 de 1930, Artículo 26). Previo a esta inscripción, el médico y en el caso de defunción fetal la matrona, debe llenar el "Certificado de defunción y estadística de mortalidad fetal tardía”, el cual será posteriormente presentado en el Servicio de Registro Civil e Identificación por cualquier persona que pueda proporcionar los datos e identificación del fallecido según requerimiento del oficial civil.

El Certificado de defunción y estadística de mortalidad fetal tardía tiene un papel clave en la generación de estadísticas de mortalidad y causas de muerte. Estas son estadísticas vitales que constituyen valiosos indicadores de salud de la población, los que permiten realizar monitoreos y seguimientos desde diferentes aspectos.

Existe un convenio entre el Ministerio de Salud (MINSAL) y el Servicio de Registro Civil e Identificación enviando este último, copias de todos los certificados de defunción que ocurren en Chile. A partir de 1997 en nuestro país se implementó la codificación de las causas de muerte con la revisión de la "Clasificación internacional de enfermedades y problemas relacionados con la salud, CIE-10". Su código alfanumérico per- mite disponer de categorías y subcategorías, lo cual permite que los diagnósticos detallados puedan traducirse en estadísticas, y finalmente en un mejor conocimiento de las causas de defunción, ya que a cada enfermedad se le asigna un código propio.

Los certificados de defunción, una vez ingresados a Estadísticas del MINSAL, son codificados según causas asociadas a SIDA por la CIE10 , incluyendo enfermedades marcadoras y enfermedades que, sin serlas, son sugerentes de infección por VIH/SIDA, lo cual finalmente se comprueba mediante otras variables asociadas, como registro ISP, sexo, edad, comuna de residencia, escolaridad, ocupación, etc, en el caso de estar previamente notificado. El proceso continúa con el envío de copias de estos certificados a CONASIDA, donde se verifica la condición de Notificado en la base de Registro Nacional de infección por VIH/SIDA.

El análisis de los certificados de defunción permite realizar estimaciones de la sensibilidad del sistema de vigilancia pasiva de SIDA, es decir, la probabilidad de que un caso sea notificado, y su contraparte, la subnotificación en infección por VIH/SIDA, es decir, quienes teniendo infección por VIH o SIDA, nunca fueron notificadas.

De acuerdo al proceso de revisión de los certificados de defunción iniciado en 1991, se estimó la sensibilidad en $75 \%$ en 2003 , es decir, 
la subnotificación alcanzó a 25,0\%. Sin embargo, se ha producido un aumento respecto al año anterior, ya que el 2002 se había encontrado una sensibilidad del sistema de $72,5 \%$ con una subnotificación de 27,5\%. Los cambios podrían ser explicados por mejorías en el registro de las causas de muerte en los certificados de defunción por parte de los profesionales médicos ${ }^{1}$ producto de la disminución de la estigmatización de los afectados, a mejorías en Estadísticas del MINSAL y a aumento del acceso a terapia antiretroviral (TARV) que exige la notificación del caso.

Se revisaron los certificados de defunción No Notificados y recolectados en el Departamento de Estadísticas del MINSAL mediante el convenio con el Servicio de Registro Civil. Se incluyeron los certificados desde el año 1997 al 2003, lo que representa un total de 492. Los certificados correspondientes al año 1999 no se recibieron y en el 2000 sólo se dispuso de una planilla desde donde se pudo obtener el sexo y la edad.

Esta revisión permitió caracterizar a los fallecidos en términos de sexo, edad, educación, ocupación, comuna de residencia en términos de ruralidad y sistema de salud al cual pertenecían, lo que permite establecer una comparación entre años de estas variables.

\section{Defunciones por año}

El gráfico 1 muestra el número de defunciones ocurridas en el país entre los años 1997 y 2003 que fueron Notificadas y No Notificadas al
Sistema de Vigilancia de infección por VIH/SIDA.

El promedio anual entre 1997-2003 de defunciones No Notificadas aumentó en 15,8\% mientras las Notificadas lo hicieron sólo en $1,82 \%$.

\section{Sexo}

En las Defunciones Notificadas la distribución porcentual por sexo se mantuvo relativamente estable desde 1997 al 2003 (Tabla 1 y gráficos 2 y 3) y se observó durante todo el período que los hombres representaban cerca del $85 \%$ de las defunciones.

El número de casos totales para el período en hombres fue de 2.207 y representaba el $86,8 \%$. En mujeres el número de defunciones fue de 335 $(13,2 \%)$. Sin embargo, se registró en las defunciones según sexo un incremento porcentual promedio $1997-2003$ de $0,63 \%$ en hombres y $4,2 \%$ en mujeres.

En las Defunciones No Notificadas la distribución porcentual por sexo se mantuvo relativamente estable desde 1997 al 2003 (Tabla 1 y gráficos 2 y 3), observándose durante todo el período que sobre $80 \%$ de las defunciones ocurrieron en hombres.

El número de casos totales para el período en hombres fue de 418 (85,0\%). En mujeres el número de defunciones fue de $74(15,0 \%)$. Sin embargo, se registró en las defunciones según sexo un incremento porcentual promedio 19972003 de $18,1 \%$ en hombres y $7,8 \%$ en mujeres.

Por lo tanto, en ambos casos el sexo masculino acumuló sobre $80 \%$ de los decesos.

Tabla 1. Número de casos y porcentaje de defunciones, notificadas y no notificadas, según sexo y años. Chile, 1997-2003

\begin{tabular}{lcccccccccccccc}
\hline & $\mathbf{1 9 9 7}$ & $\mathbf{\%}$ & $\mathbf{1 9 9 8}$ & $\mathbf{\%}$ & $\mathbf{2 0 0 0}$ & $\mathbf{\%}$ & $\mathbf{2 0 0 1}$ & $\boldsymbol{\%}$ & $\mathbf{2 0 0 2}$ & $\boldsymbol{\%}$ & $\mathbf{2 0 0 3}$ & $\boldsymbol{\%}$ & Total & $\boldsymbol{\%}$ \\
\hline Hombre notificado & 378 & 88,7 & 349 & 89,7 & 217 & 84,4 & 387 & 85,8 & 316 & 86,1 & 296 & 85,1 & 2.207 & 86,8 \\
Mujer notificada & 48 & 11,3 & 40 & 10,3 & 40 & 15,6 & 64 & 14,2 & 51 & 13,9 & 52 & 14,9 & 335 & 13,2 \\
$\begin{array}{l}\text { Total notificado } \\
426\end{array}$ & 100,0 & 389 & 100,0 & 257 & 100,0 & 451 & 100,0 & 367 & 100,0 & 348 & 100,0 & 2.542 & 100,0 \\
$\begin{array}{l}\text { Hombre no } \\
\text { notificado }\end{array}$ & 44 & 81,5 & 49 & 83,1 & 89 & 89,0 & 67 & 83,8 & 90 & 84,9 & 79 & 84,9 & 418 & 85,0 \\
$\begin{array}{l}\text { Mujer no } \\
\text { notificada }\end{array}$ & 10 & 18,5 & 10 & 16,9 & 11 & 11,0 & 13 & 16,2 & 16 & 15,1 & 14 & 15,1 & 74 & 15,0 \\
Total no notificado & 54 & 100,0 & 59 & 100,0 & 100 & 100,0 & 80 & 100,0 & 106 & 100,0 & 93 & 100,0 & 492 & 100,0 \\
\hline
\end{tabular}

${ }^{1}$ Comunicación personal obtenida del Comité Científico Asesor. Santiago. Octubre 2003. 


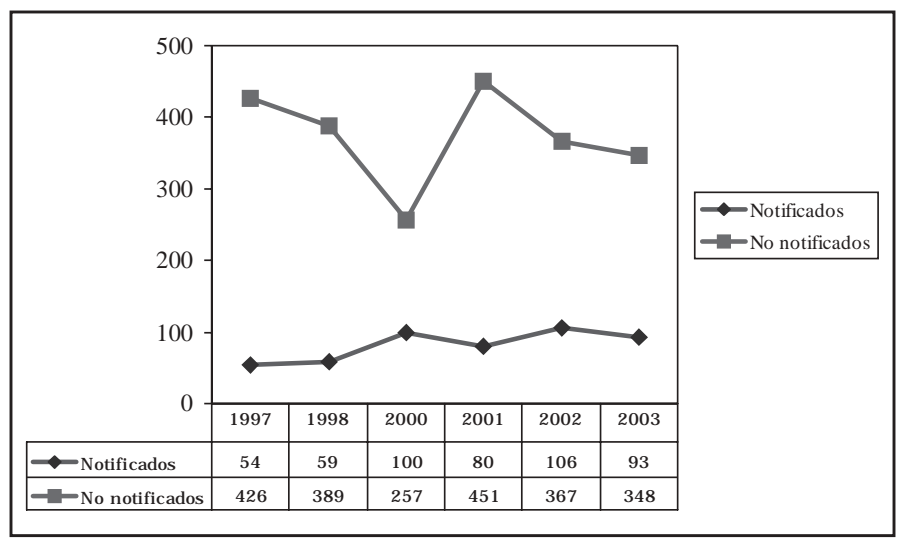

Gráfico 1. Número de defunciones notificadas y no notificadas. Ambos sexos. Chile, 1997-2003.

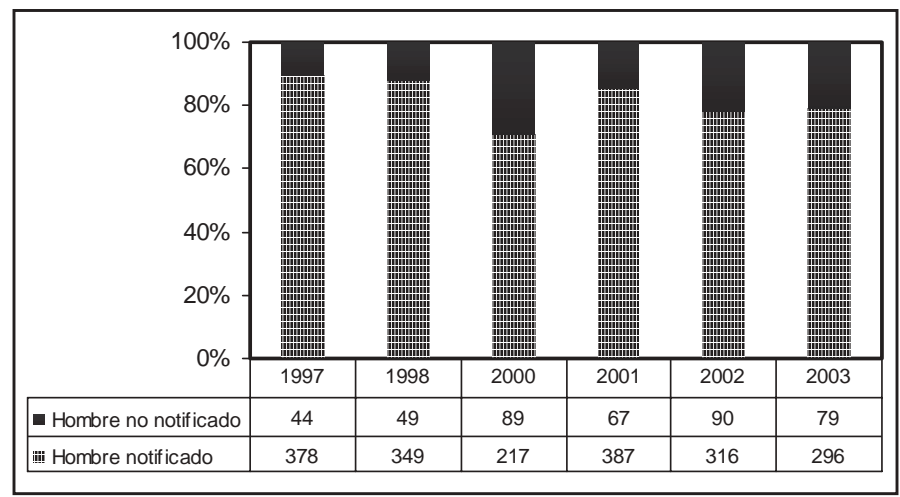

Gráfico 2. Distribución porcentual de hombres fallecidos, notificados y no notificados, según año. Chile, 1997-2003.

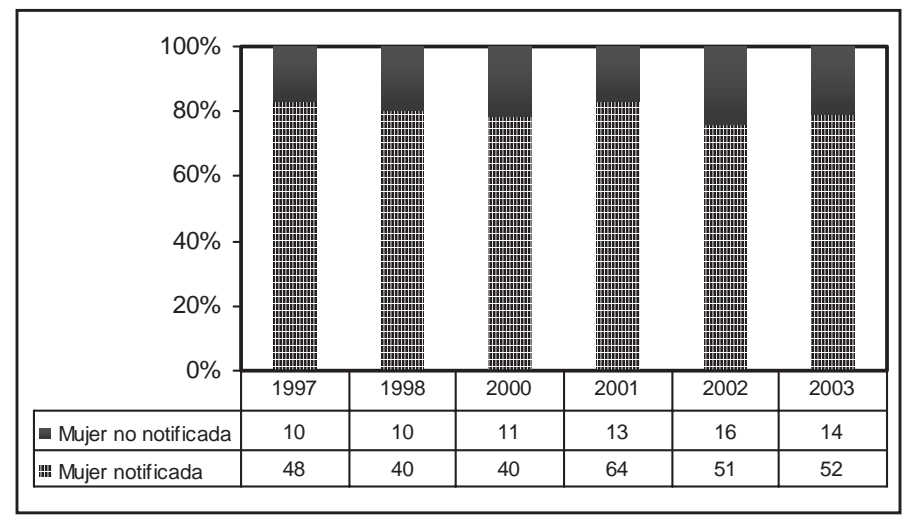

Gráfico 3. Distribución porcentual de mujeres fallecidas, notificadas y no notificadas, según año. Chile, 1997-2003.

\section{Edad}

La edad de defunción en los Notificados desde 1987 a 1999 mayoritariamente correspondió al grupo de 21 a 30 años y a partir del 2000 se desplazó al grupo etario de 31 a 40 años, invirtiéndose la proporción. El tercer grupo fue el de 41 a 50 años, manteniendo esta posición durante todo el período. Los grupos con menor frecuencia a lo largo de los años fueron los < 20 años y los >60 años (Tabla 2 y gráfico 4 ).

$\mathrm{Al}$ analizar las edades de los fallecidos No Notificados el mayor porcentaje se observó en el grupo etario 31 a 40 años $(34,6 \%)$ lo cual fue sostenido en el período 1997-2003, el segundo grupo en importancia estuvo representado por el estrato 41 a 50 años $(19,6 \%)$, estrechamente seguido por el grupo 21 a 30 años $(18,6 \%)$. Las defunciones No Notificadas, según edad al momento de fallecer, fueron más frecuentes en el grupo de 31 a 40 años, lo cual se sostuvo en el período 1997-2003 y se corresponden con las edades afectadas en la epidemia (Tabla 2, gráfico 5).

En ambos casos, ya sean Defunciones Notificadas y No Notificadas, el grupo afectado correspondió a adultos jóvenes. Sin embargo, a partir del 2000 en ambas situaciones se coincide que el estrato etario con mayor frecuencia fue el de 31 a 40 años.

\section{Educación}

Las personas fallecidas Notificadas durante 1997 al 2003 habían alcanzado en su mayoría educación media $(46,9 \%)$ y luego básica/primaria $(32,0 \%)$. El $12,3 \%$ restante declaraba nivel de educación superior (Tabla 3 y gráfico 6).

Para el período 1997-2003, los fallecidos No Notificados mayoritariamente alcanzaban el nivel educacional medio $(41,33 \%)$ y en segundo lugar el básico/primario (34,95\%). El nivel superior representaba cerca 
Tabla 2. Número de casos y porcentaje de defunciones notificadas según edad y año de defunción. Ambos sexos. Chile, 1997-2003

\begin{tabular}{|c|c|c|c|c|c|c|c|c|c|c|c|c|c|c|}
\hline Edad (años) & 1997 & $\%$ & 1998 & $\%$ & 2000 & $\%$ & 2001 & $\%$ & 2002 & $\%$ & 2003 & $\%$ & Total & $\%$ \\
\hline & $\mathbf{N}$ & $\mathbf{o}$ & $\mathbf{t}$ & i & $\mathbf{f}$ & $\mathbf{i}$ & & c & $\mathbf{a}$ & d & $\mathbf{o}$ & $\mathbf{s}$ & & \\
\hline$<20$ & 13 & 3,1 & 12 & 3,1 & 13 & 5,1 & 21 & 4,7 & 16 & 4,4 & 18 & 5,2 & 93 & 4,2 \\
\hline $21-$ & 149 & 35,2 & 141 & 36,4 & 81 & 31,6 & 137 & 30,4 & 97 & 26,6 & 89 & 25,6 & 694 & 31,1 \\
\hline $31-40$ & 148 & 35,0 & 127 & 32,8 & 91 & 35,5 & 145 & 32,2 & 142 & 38,9 & 116 & 33,4 & 769 & 34,5 \\
\hline $41-50$ & 70 & 16,5 & 64 & 16,5 & 40 & 15,6 & 93 & 20,7 & 71 & 19,5 & 64 & 18,4 & 402 & 18,0 \\
\hline $51-60$ & 31 & 7,3 & 34 & 8,8 & 20 & 7,8 & 40 & 8,9 & 31 & 8,5 & 38 & 11,0 & 194 & 8,7 \\
\hline$>61$ & 12 & 2,8 & 9 & 2,3 & 11 & 4,3 & 14 & 3,1 & 8 & 2,2 & 22 & 6,3 & 76 & 3,4 \\
\hline \multirow[t]{2}{*}{ Total } & 423 & 100,0 & 387 & 100,0 & 256 & 100,0 & 450 & 100,0 & 365 & 100,0 & 347 & 100,0 & 2.228 & 100,0 \\
\hline & $\mathbf{N}$ & $\mathbf{o}$ & & $\mathbf{N}$ & 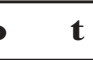 & i & $\mathbf{f}$ & i & c & $\mathbf{a}$ & o & $\mathbf{s}$ & & \\
\hline$<20$ & 3 & 5,6 & 2 & 3,4 & 2 & 2,0 & 2 & 2,5 & 1 & 1,0 & 1 & 1,1 & 11 & 2,2 \\
\hline $21-30$ & 14 & 25,9 & 12 & 20,3 & 13 & 13,0 & 13 & 16,3 & 24 & 23,3 & 15 & 16,1 & 91 & 18,6 \\
\hline $31-40$ & 19 & 35,2 & 22 & 37,3 & 32 & 32,0 & 26 & 32,5 & 36 & 35,0 & 34 & 36,6 & 169 & 34,6 \\
\hline $41-50$ & 3 & 5,6 & 10 & 16,9 & 29 & 29,0 & 13 & 16,3 & 20 & 19,4 & 21 & 22,6 & 96 & 19,6 \\
\hline $51-60$ & 8 & 14,8 & 5 & 8,5 & 18 & 18,0 & 14 & 17,5 & 15 & 14,6 & 12 & 12,9 & 72 & 14,7 \\
\hline$>61$ & 7 & 13,0 & 8 & 13,6 & 6 & 6,0 & 12 & 15,0 & 7 & 6,8 & 10 & 10,8 & 50 & 10,2 \\
\hline Total & 54 & 100,0 & 59 & 100,0 & 100 & 100,0 & 80 & 100,0 & 103 & 100,0 & 93 & 100,0 & 489 & 100,0 \\
\hline
\end{tabular}

de $11 \%$; este valor, a pesar de estar cerca de cinco puntos bajo el promedio nacional según Censo 2002, es considerado bueno.

Sin embargo, el elevado porcentaje en educación básica nos muestra que estos fallecidos se encontraban en una situación más pauperizada (Tabla 3 y gráfico 7).

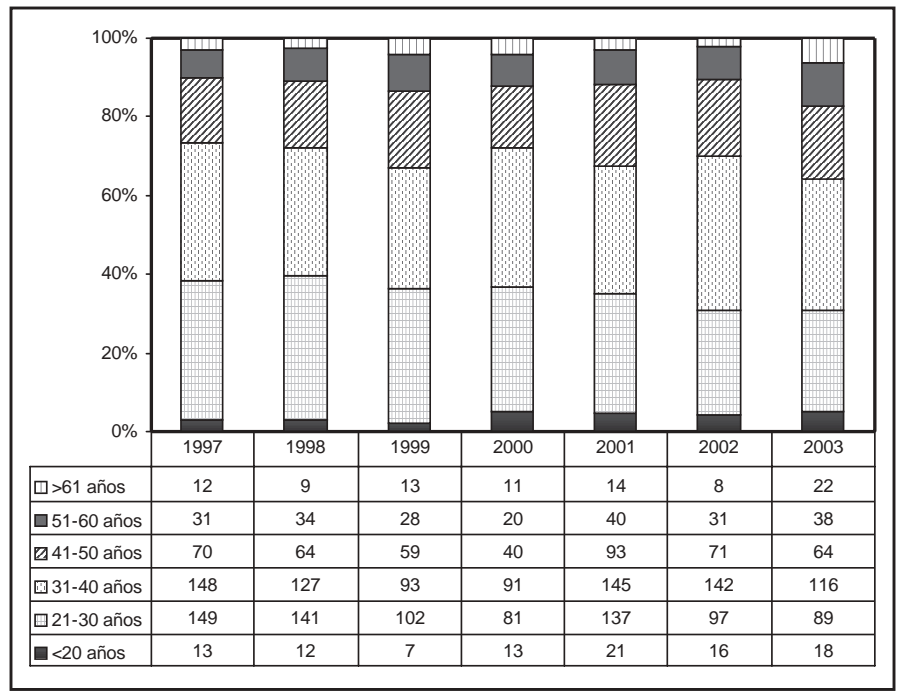

Gráfico 4. Distribución porcentual de edad en defunciones notificadas. Ambos sexos. Chile. 1997-2003.
Al comparar los niveles educaciones entre los fallecidos Notificados y No Notificados se logró evidenciar escasas diferencias, el nivel básico en ambos se mantuvo sobre $30 \%$, con una diferencia de 3 puntos porcentuales a favor de los Notificados. La categoría secundario no se registró en las defunciones Notificadas pero, al sumarla al nivel medio se obtuvo $50,3 \%$ lo cual supera levemente a las defunciones No Notificadas en $3,4 \%$. La categoría superior fue levemente inferior en 1,3\% en los fallecidos Notificados con respecto a los No Notificados.

Los gráficos 6 y 7 muestran cómo la tendencia en estos años fue relativamente estable en ambos casos.

$\mathrm{Al}$ analizar el nivel educacional según sexo en los Notificados se apreció cómo en las mujeres éste se desplazó hacia niveles más bajos, siendo de mayores frecuencias porcentuales y predominio dentro del período el básico/primario. En hombres el nivel medio fue más frecuente (Tabla 4). Por lo tanto, la mujer aparecía más desprotegida desde el punto de vista educacional. 


\begin{tabular}{|c|c|c|c|c|c|}
\hline $80 \%$ & & & & & \\
\hline $60 \%$ & & & & & \\
\hline $40 \%$ & & & & & 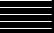 \\
\hline $20 \%$ & & 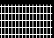 & 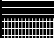 & 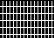 & \\
\hline $0 \%$ & 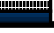 & 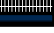 & 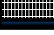 & 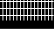 & 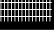 \\
\hline & 1997 & 1998 & 2001 & 2002 & 2003 \\
\hline$\boxplus>61$ años & 7 & 8 & 12 & 7 & 10 \\
\hline 口 51-60 años & 8 & 5 & 14 & 15 & 12 \\
\hline ๒ 41-50 años & 3 & 10 & 13 & 20 & 21 \\
\hline घ 31-40 años & 19 & 22 & 26 & 36 & 34 \\
\hline 田 21-30 años & 14 & 12 & 13 & 24 & 15 \\
\hline
\end{tabular}

Gráfico 5. Distribución porcentual de edad en defunciones no notificadas. Ambos sexos. Chile, 1997-2003.

El nivel educacional en No Notificados nos mostró en las mujeres un desplazamiento hacia niveles más bajos, siendo el básico/primario el más frecuente y predominante dentro del período. En hombres el nivel medio fue el más común durante todos los años (Tabla 5).

Tanto en fallecidas Notificadas como No Notificadas se alcanzaron valores cercanos e incluso superiores al $50 \%$ de educación básica/primaria.

\section{Ocupación}

Al evaluar el tipo de ocupación por sexos desde los certificados de Defunciones Notificadas, vemos que, en el caso de los hombres, la mayoría porcentual se ubicaba en el nivel operarios con valores desde 51,9 a $60,5 \%$, siendo el promedio anual $56 \%$. Si bien existía alrededor de $8,1 \%$ de profesionales en el caso de los hombres, la mayoría de los fallecidos tuvo ocupaciones relacionadas con niveles más pauperizados de la población (Tabla 6), entre ellos obreros $(7,5 \%)$, peluqueros $(5,5 \%)$, comerciantes $(4,4 \%)$, vendedores $(4,3 \%)$, choferes $(3,6 \%)$, empleados $(3,2 \%)$, operadores $(3,1 \%)$ y trabajadores sexuales $(2,4 \%)$. Igual situación se vio en las mujeres, incluso siendo más marcada la contribución de la categoría otros, explicada por un porcentaje importante de mujeres fallecidas que eran dueñas de casa $(47,8 \%)$, asesoras del hogar $(10,7 \%)$ y trabajadoras sexuales $(6,9 \%)$ (Tabla 7).

Tabla 3. Distribución de personas fallecidas, notificadas y no notificadas, según nivel educacional. Ambos sexos. Chile, 1997-2003

\begin{tabular}{lrrrrrrr}
\hline Nivel educacional & $\mathbf{1 9 9 7}$ & $\mathbf{1 9 9 8}$ & $\mathbf{2 0 0 1}$ & $\mathbf{2 0 0 2}$ & $\mathbf{2 0 0 3}$ & TOTAL & \% \\
\hline Notificadas & & & & & & & \\
Básico/primario & 129 & 120 & 154 & 114 & 111 & 628 & 33,8 \\
Medio & 211 & 180 & 208 & 179 & 142 & 920 & 49,5 \\
Superior & 58 & 58 & 54 & 49 & 41 & 260 & 14,0 \\
Ninguno & 10 & 8 & 13 & 9 & 12 & 52 & 2,8 \\
Total & 408 & 366 & 429 & 351 & 306 & 1.860 & 100,00 \\
& & & & & & & \\
No notificadas & 17 & 20 & 30 & 38 & 32 & 137 & 34,95 \\
Básico/primario & 22 & 23 & 32 & 47 & 38 & 162 & 41,33 \\
Medio & 6 & 9 & 4 & 5 & 11 & 35 & 8,93 \\
Secundario & 6 & 3 & 10 & 14 & 10 & 43 & 10,97 \\
Superior & 0 & 2 & 4 & 2 & 2 & 10 & 2,55 \\
Ninguno & 54 & 59 & 80 & 106 & 93 & 392 & 100,00 \\
Total & & & & & & & \\
\hline
\end{tabular}




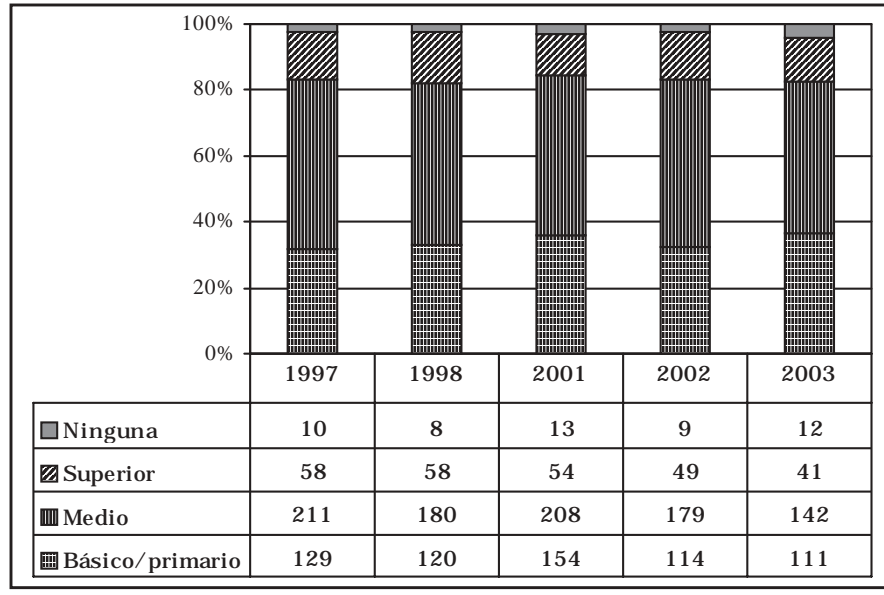

Gráfico 6. Distribución porcentual de personas fallecidas notificadas, según nivel de escolaridad. Ambos sexos. Chile, 1997-2003.

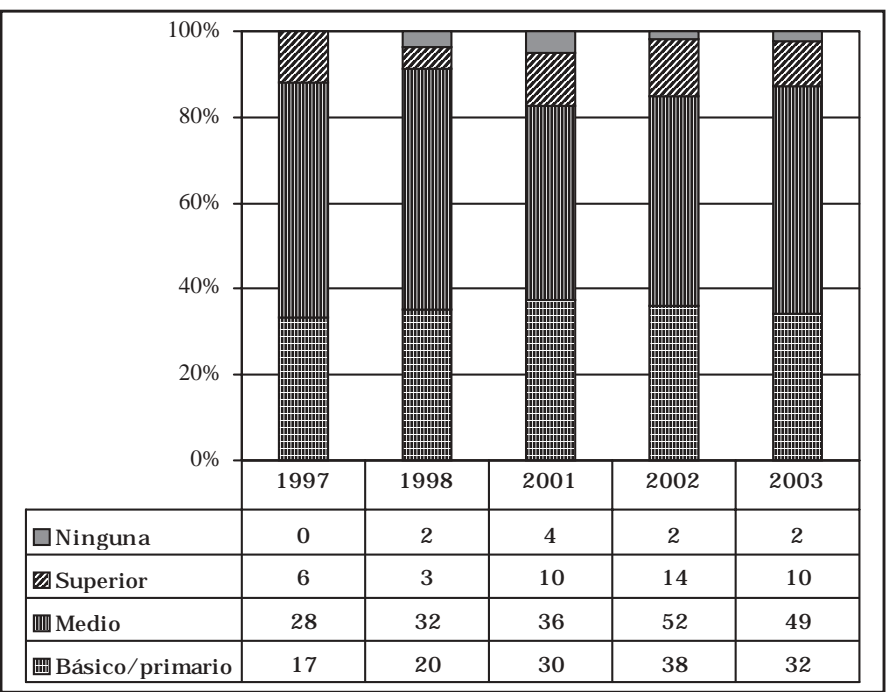

Gráfico 7. Distribución porcentual de personas fallecidas no notificadas, según nivel de escolaridad. Ambos sexos. Chile, 1997-2003.
Al evaluar el tipo de ocupación por sexos en Defunciones No Notificadas, en el caso de los hombres la mayoría porcentual se ubicaba en el nivel operarios y otros, incluyendo este último a estudiantes, pensionados y cesantes. En este análisis dentro del grupo otros sobresalía la categoría pensionados(as). Si bien existía alrededor de $11 \%$ de profesionales entre los hombres, la mayoría de los fallecidos tuvo ocupaciones relacionadas con niveles más pauperizados de la población (Tabla 6). Igual situación se vio en las mujeres, incluso siendo más marcada la contribución de la categoría otros, explicado por un porcentaje importante de mujeres fallecidas y también afectadas por la epidemia que eran dueñas de casa $(48,4 \%)$, pensionadas y lo jubiladas $(21,9 \%)$ y asesoras del hogar $(4,7 \%)$ (Tabla 7 ).

En las mujeres, durante todos los años se registró una mayor frecuencia en el grupo de otros, el cual incluye a las dueñas de casa y estudiantes. Durante 1997, del total de 54 defunciones por SIDA no notificadas, 3 correspondieron a dueñas de casa $(5,6 \%)$ y ninguna a estudiante. En 1998 las dueñas de casa fallecidas fueron 3 , correspondiendo a $5,1 \%$ del total para el año, y los estudiantes fallecidos fueron 2 , al-

Tabla 4. Distribución porcentual de hombres fallecidos, notificados y no notificados, según nivel educacional. Chile, 1997-2003

\begin{tabular}{lrrrrr}
\hline Nivel educacional & $\mathbf{1 9 9 7}$ & $\mathbf{1 9 9 8}$ & $\mathbf{2 0 0 1}$ & $\mathbf{2 0 0 2}$ & $\mathbf{2 0 0 3}$ \\
\hline Notificados & & & & & \\
Básico/primario & 27,2 & 32,1 & 32,9 & 29,8 & 32,7 \\
Medio & 52,1 & 49,4 & 51,4 & 52,3 & 48,5 \\
Superior & 15,3 & 17,0 & 13,9 & 15,6 & 15,0 \\
Ninguno & 1,39 & 1,9 & 2,3 & 3,8 \\
No notificados & & & & & \\
Básico/primario & 22,7 & 36,7 & 35,8 & 34,4 & 31,6 \\
Medio & 47,7 & 40,8 & 40,3 & 44,4 & 43,0 \\
Secundario & 13,6 & 14,3 & 4,5 & 5,6 & 12,7 \\
Superior & 13,6 & 6,1 & 13,4 & 14,4 & 11,4 \\
No aplica & 2,3 & 0,00 & 0,0 & 0,0 & 0,0 \\
Ninguno & 0,00 & 2,04 & 6,0 & 1,1 & 1,3 \\
\hline
\end{tabular}


canzando 3,4\%. Para los años 1999 y 2000 no se dispuso de esta información. Durante el 2001 se registraron 11 defunciones en dueñas de casa, lo que representó para el total del año $13,8 \%$ y ningún estudiante. En el 2002 las dueñas de casa fallecidas no notificadas fueron $7(6,6 \%)$ y 6 eran estudiantes $(5,7 \%)$. Finalmente, en el 2003 las dueñas de casa fallecidas fueron $7(7,5 \%)$, mientras que estudiante era sólo $1(1,1 \%)$.

En ambas situaciones, ya sean Defunciones
Notificadas y No Notificadas, los grupos más afectados fueron los operarios en el caso de hombres y dueñas de casa en mujeres.

\section{Ruralidad}

Las comunas de residencia de los fallecidos fueron agrupadas en quintiles de acuerdo a la clasificación utilizada en las publicaciones del

Tabla 5. Distribución porcentual de mujeres fallecidas, notificadas y no notificadas, según nivel educacional. Chile, 1997-2003

\begin{tabular}{lccccc}
\hline Nivel educacional & $\mathbf{1 9 9 7}$ & $\mathbf{1 9 9 8}$ & $\mathbf{2 0 0 1}$ & $\mathbf{2 0 0 2}$ & $\mathbf{2 0 0 3}$ \\
\hline Notificadas & & & & & \\
Básico/primario & 54,2 & 35,0 & 54,1 & 48,9 & 56,5 \\
Medio & 29.2 & 42.5 & 31,1 & 42,9 & 34,8 \\
Superior & 6.3 & 5.0 & 4,9 & 4,1 & 4,3 \\
Ninguno & 10.4 & 7.5 & 9,8 & 4,1 & 4,3 \\
& & & & & \\
No notificadas & & & 46,1 & 43,7 & 50,0 \\
Básico/primario & 70,0 & 20,00 & 38,5 & 43,7 & 28,6 \\
Medio & 10,0 & 30,0 & 0,05 & 0,0 & 7,1 \\
Secundario & 0,0 & 20,0 & 7,69 & 6,2 & 7,1 \\
Superior & 0,0 & 0,0 & 0.7 & 0,0 & 0.0 \\
No aplica & 20,0 & 20,0 & 0,0 & 6,2 & 7,1 \\
Ninguno & 0,0 & 10,0 & & & \\
\hline
\end{tabular}

Tabla 6. Distribución porcentual defunciones, notificadas y no notificadas, en hombres, según nivel de ocupación. Chile, 1997-2003

\begin{tabular}{lrrrrr}
\hline Nivel & $\mathbf{1 9 9 7}$ & $\mathbf{1 9 9 8}$ & $\mathbf{2 0 0 1}$ & $\mathbf{2 0 0 2}$ & $\mathbf{2 0 0 3}$ \\
\hline Notificados & & & & & \\
Ejecutivo & 1,6 & 0,6 & 0,5 & 0,6 & 0,3 \\
Profesional & 9,3 & 10,6 & 9,0 & 7,9 & 8,1 \\
Oficinista & 19,8 & 18,9 & 13,7 & 15,2 & 11,1 \\
Operario & 53,7 & 51,9 & 60,5 & 56,3 & 57,4 \\
Otros & 5,3 & 4,6 & 5,9 & 8,2 & 8,4 \\
S/i & 10,3 & 13,5 & 10,3 & 11,7 & 14,5 \\
Total & 100,0 & 100,0 & 100,0 & 100,0 & 100,0 \\
& & & & & \\
No notificados & & & & & \\
Ejecutivo & 0,0 & 0,0 & 0,0 & 1,1 & 0,0 \\
Profesional & 15,9 & 8,2 & 13,4 & 8,9 & 7,3 \\
Oficinista & 15,9 & 20,4 & 10,4 & 14,4 & 36,7 \\
Operario & 38,6 & 26,5 & 23,9 & 32,2 & 26,6 \\
Otros & 27,3 & 45,0 & 41,8 & 25,6 & 11,4 \\
No aplica & 2,3 & 0,0 & 9,0 & 16,7 & 11,4 \\
S/i & 0,0 & 0,0 & 1,5 & 1,1 & 100,0 \\
Total & 100,0 & 100,0 & 100,0 & 100,0 & \\
\hline
\end{tabular}


Boletín Epidemiológico de VIH/SIDA, según los siguientes porcentajes de ruralidad:

$$
\begin{aligned}
& \mathrm{Q} 1=<20 \% \\
& \mathrm{Q} 2=20-39 \% \\
& \mathrm{Q} 3=40-59 \% \\
& \mathrm{Q} 4=60-80 \% \\
& \mathrm{Q} 5=>80 \%
\end{aligned}
$$

Con esta clasificación logramos ver que la mayoría porcentual durante todos los años se concentró en el Q1, es decir, los fallecidos tenían su residencia en sectores con menos del $20 \%$ de ruralidad, por lo tanto, residían en áreas urbanas.

En las Defunciones Notificadas desde 1997 al 2003, la mayoría de los fallecidos residían en comunas urbanas. Sin embargo, a lo largo de los años se produjo una disminución de esta categoría que en promedio alcanzó a $0,8 \%$ y con una fuerte disminución entre los años 2002 y 2003 de $11,3 \%$. La segunda categoría (Q2) aumentó entre 1997 y 2003 en 31,5\%. Los grupos Q3 y Q4 aumentaron en $123,6 \%$ y $127,6 \%$, respectivamente. Finalmente la categoría Q5, en igual período aumentó en 45,8\% (Tabla 8). Al producirse una disminución en las categorías más urbanas se ha generado un desplazamiento hacia el aumento de defunciones en las comunas con mayores porcentajes de ruralidad.

En las Defunciones No Notificadas desde 1997 al 2003, la mayoría de los fallecidos residían en comunas urbanas. A lo largo del período se produjo un incremento promedio de $13,2 \%$. La categoría Q2 aumentó entre 1997 y 2003 en $103 \%$. Los grupos Q3 y Q4 aumentaron en $12,5 \%$ y $37,5 \%$, respectivamente. Finalmente la categoría Q5, en igual período disminuyó en $25 \%$ (Tabla 8).

Al comparar el porcentaje de ruralidad entre Defunciones Notificadas y No Notificadas, hubo diferencias dado en la primera por la tendencia a un incremento en las comunas con porcentajes mayores de ruralidad, a diferencia de los No Notificados donde aumentó en las comunas más urbanas.

\section{Índice de desarrollo humano}

Se realizó a la vez, un ordenamiento de las comunas declaradas como residencia en los certificados de defunción según el Índice de Desarrollo Humano (IDH). Se entiende por Desarrollo Humano el proceso de ampliación de las capaci-

Tabla 7. Distribución porcentual defunciones, notificadas y no notificadas, en mujeres, según nivel de ocupación. Chile, 1997-2003

\begin{tabular}{lrrrrr}
\hline Nivel & $\mathbf{1 9 9 7}$ & $\mathbf{1 9 9 8}$ & $\mathbf{2 0 0 1}$ & $\mathbf{2 0 0 2}$ & $\mathbf{2 0 0 3}$ \\
\hline Notificadas & & & & & \\
Ejecutivo & 0 & $\mathrm{O}$ & $\mathrm{O}$ & 0 & 0 \\
Profesional & 2,1 & 2,5 & 1,6 & 3,9 & 1,9 \\
Oficinista & 8,3 & 7,5 & 9,4 & 9,8 & 1,9 \\
Operario & 35,4 & 27,5 & 40,6 & 23,5 & 36,5 \\
Otros & 54,2 & 52,5 & 45,3 & 54,9 & 53,8 \\
S/i & 0 & 10,0 & 3,1 & 7,8 & 5,8 \\
Total & 100,0 & 100,0 & 100,0 & 100,0 \\
& & & & \\
No notificadas & & & & & \\
Ejecutivo & 0,0 & 0,0 & 0,0 & 0,0 & 0,0 \\
Profesional & 0,0 & 0,0 & 0,0 & 6,2 & 7,1 \\
Oficinista & 0,0 & 20,0 & 0,0 & 6,2 & 0,0 \\
Operario & 10,0 & 10,0 & 0,0 & 12,5 & 21,4 \\
Otros & 70,0 & 50,0 & 92,3 & 68,7 & 71,4 \\
No aplica & 20,0 & 20,0 & 7,7 & 6,2 & 0,0 \\
S/i & 0,0 & 0,0 & 0,0 & 0,0 & 0,0 \\
Total & 100,0 & 100,0 & 100,0 & 100,0 & 100,0 \\
\hline
\end{tabular}


Tabla 8. Distribución porcentual según nivel de ruralidad en defunciones notificadas y no notificadas acumuladas. Ambos sexos. Chile, 1997-2003

\begin{tabular}{lrrrrr}
\hline \% ruralidad & $\mathbf{1 9 9 7}$ & $\mathbf{1 9 9 8}$ & $\mathbf{2 0 0 1}$ & $\mathbf{2 0 0 2}$ & $\mathbf{2 0 0 3}$ \\
\hline Notificadas & & & & & \\
$<20$ & 93,1 & 93,0 & 89,7 & 5,7 & 6,7 \\
$40-59$ & 1,9 & 1,0 & 1,8 & 1,1 & 3,2 \\
$60-80$ & 1,2 & 1,6 & 1,8 & 0,3 & 2,6 \\
$>80$ & 0,7 & 0 & 0,4 & 1,1 & 0,9 \\
& & & & & \\
No notificadas & & & & 83,0 & 89,2 \\
$<20$ & 98,1 & 88,1 & 90,0 & 13,2 & 7,5 \\
$20-39$ & 1,9 & 5,1 & 5,0 & 2,8 & 0 \\
$40-59$ & 0 & 3,4 & 1,3 & 0,9 & 3,2 \\
$60-80$ & 0 & 3,4 & 1,3 & 0 & 0 \\
$>80$ & 0 & 0 & 2,5 & & \\
\hline
\end{tabular}

dades de las personas. Las capacidades de las personas suelen ser múltiples y cambiar en el tiempo. Existen, sin embargo, algunas condiciones básicas y que son comunes a todas las sociedades y en todo tiempo como son: tener una vida larga y sana; poseer los conocimientos necesarios para comprender y relacionarse con el entorno social y poseer los ingresos suficientes para acceder a un nivel de vida decente. Estas son las dimensiones que, en base a diferentes indicadores, se pretende recoger mediante el IDH. En lo metodológico muestra cuán lejos o cerca se está de una meta ideal.

En el análisis de los certificados de Defunción Notificados se registraron 203 comunas de un total de 342 comunas nacionales, representando $59,4 \%$. Estas comunas notificaron un total de 4.091 defunciones y al ser estratificadas en los intervalos de IDH 1-133, 134-233 y 234-333 dieron porcentajes de participación dentro del total de fallecidos no notificados de 52,7, 22,7 y 23,2\% respectivamente (Tabla 9).

En el análisis de los certificados de Defunción No Notificados se registraron 103 comunas de un total de 342 comunas nacionales, representando $30,1 \%$. Estas comunas notificaron en total 404 defunciones y al ser estratificadas en los intervalos de IDH 1-133, 134-233 y 234-333 dieron porcentajes de participación dentro del total de fallecidos no notificados de 86,$1 ; 10,4$ y $3,5 \%$ respectivamente, es decir, la mayoría logró un índice de desarrollo humano adecuado $(86,1 \%)$ y la minoría se ubicó en el nivel inferior $(3,5 \%)$ (Tabla 10).
Tabla 9. Número de casos de infección por VIH/ SIDA en comunas, según nivel de logro en el índice de desarrollo humano. Defunciones notificadas. Ambos sexos. Chile, 1997-2003

\begin{tabular}{|c|c|c|c|}
\hline IDH* & Comunas & Casos & Orden IDH \\
\hline 1 & Antofagasta & 75 & 1 \\
\hline 1 & Arica & 55 & 2 \\
\hline 1 & Aysén & 3 & 3 \\
\hline 1 & Buin & 7 & 4 \\
\hline 1 & Calama & 24 & 5 \\
\hline 1 & Caldera & 1 & 6 \\
\hline 1 & Calera & 16 & 7 \\
\hline 1 & Calera de Tango & 2 & 8 \\
\hline 1 & Cartagena & 8 & 9 \\
\hline 1 & Casablanca & 6 & 10 \\
\hline 1 & Castro & 3 & 11 \\
\hline 1 & Cerrillos & 16 & 12 \\
\hline 1 & Chillán & 34 & 13 \\
\hline 1 & Coyhaique & 8 & 14 \\
\hline 1 & Colina & 11 & 15 \\
\hline 1 & Concepción & 49 & 16 \\
\hline 1 & Conchalí & 132 & 17 \\
\hline 1 & Copiapó & 8 & 18 \\
\hline 1 & Coquimbo & 28 & 19 \\
\hline 1 & Curacaví & 1 & 20 \\
\hline 1 & Curicó & 7 & 21 \\
\hline 1 & Diego de Almagro & 2 & 22 \\
\hline 1 & El Bosque & 66 & 23 \\
\hline
\end{tabular}


Tabla 9. (Continuación 9)

\begin{tabular}{|c|c|c|c|c|c|c|c|}
\hline IDH** & Comunas & Casos & Orden IDH & IDH* & Comunas & Casos & Orden IDH \\
\hline 1 & El Tabo & 2 & 24 & 1 & Quillota & 29 & 77 \\
\hline 1 & Estación Central & 80 & 25 & 1 & Quilpue & 56 & 78 \\
\hline 1 & Huechuraba & 31 & 26 & 1 & Quinta de Tilcoco & 3 & 79 \\
\hline 1 & Illapel & 1 & 27 & 1 & Quinta Normal & 60 & 80 \\
\hline 1 & Independencia & 36 & 28 & 1 & Quintero & 10 & 81 \\
\hline 1 & Iquique & 71 & 29 & 1 & Rancagua & 34 & 82 \\
\hline 1 & La Cisterna & 51 & 30 & 1 & Recoleta & 85 & 83 \\
\hline 1 & La Cruz & 1 & 31 & 1 & Renca & 58 & 84 \\
\hline 1 & La Florida & 119 & 32 & 1 & San Antonio & 26 & 85 \\
\hline 1 & La Granja & 50 & 33 & 1 & San Bernardo & 74 & 86 \\
\hline 1 & La Ligua & 6 & 34 & 1 & San Felipe & 31 & 87 \\
\hline 1 & La Pintana & 61 & 35 & 1 & San Fernando & 3 & 88 \\
\hline 1 & La Reina & 20 & 36 & 1 & San Joaquín & 56 & 89 \\
\hline 1 & La Serena & 44 & 37 & 1 & San José de Maipo & 4 & 90 \\
\hline 1 & Las Condes & 66 & 38 & 1 & San Miguel & 62 & 91 \\
\hline 1 & Limache & 7 & 39 & 1 & San Ramón & 37 & 92 \\
\hline 1 & Llay-Llay & 3 & 40 & 1 & Santiago & 347 & 93 \\
\hline 1 & Lo Barnechea & 8 & 41 & 1 & Talagante & 7 & 94 \\
\hline 1 & Lo Prado & 52 & 42 & 1 & Talca & 62 & 95 \\
\hline 1 & Los Andes & 14 & 43 & 1 & Talcahuano & 39 & 96 \\
\hline 1 & Los Ángeles & 8 & 44 & 1 & Temuco & 26 & 97 \\
\hline 1 & Los Vilos & 3 & 45 & 1 & Tierra Amarilla & 1 & 98 \\
\hline 1 & Machalí & 1 & 46 & 1 & Til-Til & 1 & 99 \\
\hline 1 & Macul & 52 & 47 & 1 & Tocopilla & 10 & 100 \\
\hline 1 & Maipú & 88 & 48 & 1 & Valdivia & 23 & 101 \\
\hline 1 & Malloa & 1 & 49 & 1 & Vallenar & 6 & 102 \\
\hline 1 & María Elena & 1 & 50 & 1 & Valparaíso & 222 & 103 \\
\hline 1 & Maullín & 1 & 51 & 1 & Vicuna & 2 & 104 \\
\hline 1 & Mejillones & 2 & 52 & 1 & Villa Alemana & 30 & 105 \\
\hline 1 & Melipilla & 8 & 53 & 1 & Viña del Mar & 143 & 106 \\
\hline 1 & Puerto Natales & 1 & 54 & 1 & Vitacura & 14 & 107 \\
\hline 1 & Nogales & 1 & 55 & 2 & Angol & 1 & 108 \\
\hline 1 & Ñuñoa & 112 & 56 & 2 & Arauco & 7 & 109 \\
\hline 1 & Olmué & 2 & 57 & 2 & Bulnes & 1 & 110 \\
\hline 1 & Osorno & 25 & 58 & 2 & Cabildo & 1 & 111 \\
\hline 1 & Ovalle & 9 & 59 & 2 & Camina & 1 & 112 \\
\hline 1 & Paihuano & 2 & 60 & 2 & Cauquenes & 5 & 113 \\
\hline 1 & Paine & 1 & 61 & 2 & Cerro Navia & 52 & 114 \\
\hline 1 & Panquehue & 2 & 62 & 2 & Colbún & 1 & 115 \\
\hline 1 & Pedro Aguirre Cerda & 52 & 63 & 2 & Coltauco & 1 & 116 \\
\hline 1 & Peñaflor & 14 & 64 & 2 & Coronel & 18 & 117 \\
\hline 1 & Peñalolén & 73 & 65 & 2 & Corral & 2 & 118 \\
\hline 1 & Pirque & 2 & 66 & 2 & Cunco & 1 & 119 \\
\hline 1 & Placilla & 1 & 67 & 2 & Curacautín & 1 & 120 \\
\hline 1 & Providencia & 68 & 68 & 2 & El Monte & 5 & 121 \\
\hline 1 & Puchuncaví & 2 & 69 & 2 & El Quisco & 2 & 122 \\
\hline 1 & Pudahuel & 54 & 70 & 2 & Freirina & 1 & 123 \\
\hline 1 & Puente Alto & 114 & 71 & 2 & Graneros & 4 & 124 \\
\hline 1 & Puerto Montt & 15 & 72 & 2 & Huayco & 1 & 125 \\
\hline 1 & Puerto Varas & 2 & 73 & 2 & Laja & 4 & 126 \\
\hline 1 & Punta Arenas & 11 & 74 & 2 & Lampa & 1 & 127 \\
\hline 1 & Quilicura & 18 & 75 & 2 & Las Cabras & 1 & 128 \\
\hline 1 & Quillón & 1 & 76 & 2 & Lautaro & 1 & 129 \\
\hline
\end{tabular}


Tabla 9. (Continuación 9)

\begin{tabular}{|c|c|c|c|}
\hline IDH** & Comunas & Casos & Orden IDH \\
\hline 2 & Linares & 16 & 130 \\
\hline 2 & Llanquihue & 1 & 131 \\
\hline 2 & Lo Espejo & 60 & 132 \\
\hline 2 & Loncoche & 1 & 133 \\
\hline 2 & Lota & 7 & 134 \\
\hline 2 & Marchigüe & 1 & 135 \\
\hline 2 & Mostazal & 1 & 136 \\
\hline 2 & Pelarco & 2 & 137 \\
\hline 2 & Penco & 5 & 138 \\
\hline 2 & Pichidegua & 1 & 139 \\
\hline 2 & Pitrufquén & 1 & 140 \\
\hline 2 & Pozo Almonte & 2 & 141 \\
\hline 2 & Purranque & 2 & 142 \\
\hline 2 & Rengo & 3 & 143 \\
\hline 2 & Requínoa & 2 & 144 \\
\hline 2 & San Carlos & 6 & 145 \\
\hline 2 & San Pedro de Atacama & 1 & 146 \\
\hline 2 & Santa Cruz & 3 & 147 \\
\hline 2 & Santa María & 2 & 148 \\
\hline 2 & Taltal & 1 & 149 \\
\hline 2 & Teno & 1 & 150 \\
\hline 2 & Tomé & 3 & 151 \\
\hline 2 & Villarrica & 4 & 152 \\
\hline 2 & Yungay & 2 & 153 \\
\hline 3 & Cabrero & 2 & 154 \\
\hline 3 & Carahue & 1 & 155 \\
\hline 3 & Coelemu & 2 & 156 \\
\hline 3 & Coihueco & 2 & 157 \\
\hline 3 & Collipulli & 3 & 158 \\
\hline 3 & Constitución & 3 & 159 \\
\hline 3 & Curanilahue & 11 & 160 \\
\hline 3 & Curepto & 2 & 161 \\
\hline 3 & El Carmen & 1 & 162 \\
\hline 3 & Entre Lagos & 1 & 163 \\
\hline 3 & Ercilla & 2 & 164 \\
\hline 3 & Florida & 1 & 165 \\
\hline 3 & Freire & 1 & 166 \\
\hline
\end{tabular}

\begin{tabular}{|c|c|c|c|}
\hline IDH* & Comunas & Casos & Orden IDH \\
\hline 3 & Gorbea & 2 & 167 \\
\hline 3 & Hualañé & 9 & 168 \\
\hline 3 & Hualqui & 1 & 169 \\
\hline 3 & Lago Ranco & 2 & 170 \\
\hline 3 & Lebu & 6 & 171 \\
\hline 3 & Longaví & 3 & 172 \\
\hline 3 & Maule & 2 & 173 \\
\hline 3 & Molina & 4 & 174 \\
\hline 3 & Monte Patria & 1 & 175 \\
\hline 3 & Nacimiento & 1 & 176 \\
\hline 3 & Negrete & 1 & 177 \\
\hline 3 & Nueva Imperial & 4 & 178 \\
\hline 3 & Paillaco & 1 & 179 \\
\hline 3 & Panguipulli & 4 & 180 \\
\hline 3 & Parral & 3 & 181 \\
\hline 3 & Portezuelo & 1 & 182 \\
\hline 3 & Purén & 1 & 183 \\
\hline 3 & Putaendo & 1 & 184 \\
\hline 3 & Quilleco & 2 & 185 \\
\hline 3 & Quirihue & 1 & 186 \\
\hline 3 & Rauco & 1 & 187 \\
\hline 3 & Río Bueno & 2 & 188 \\
\hline 3 & Río Claro & 2 & 189 \\
\hline 3 & Sagrada Familia & 1 & 190 \\
\hline 3 & San Clemente & 4 & 191 \\
\hline 3 & San Ignacio & 2 & 192 \\
\hline 3 & San Javier & 6 & 193 \\
\hline 3 & San Juan de la Costa & 2 & 194 \\
\hline 3 & Toltén & 2 & 195 \\
\hline 3 & Traiguén & 1 & 196 \\
\hline 3 & Vichuquén & 1 & 197 \\
\hline 3 & Victoria & 7 & 198 \\
\hline 3 & Vilcún & 1 & 199 \\
\hline 3 & Yumbel & 2 & 200 \\
\hline . & Chiguayante & 3 & 201 \\
\hline . & Isla de Pascua & 1 & 202 \\
\hline . & San Pedro de la Paz & 1 & 203 \\
\hline
\end{tabular}

* comunas nuevas las cuales no alcanzaron a ser incluidas en el "estudio de desarrollo humano en las comunas de Chile. MIDEPLAN/PNUD, 2000".

** Clasificación de IDH estratificado según ranking: $1=1-133 \quad 2=134-233 \quad 3$ =234-333

\section{Sistema de previsión de salud}

Con la colaboración de la Superintendencia de ISAPRES se consultó sobre el sistema al cual pertenecían los fallecidos por SIDA en el período 2001-2003 y que no fueron notificados al Sistema de Vigilancia Epidemiológica de infección por VIH/SIDA del MINSAL.

En el 2001, de un total de 80 defunciones, 17 correspondían al sector privado (21,3\%). Du- rante el 2002 se registraron 104 defunciones; de este total, 18 estaban afiliados al sector privado $(17,3 \%)$ y finalmente, el 2003 de un total de 93 defunciones no notificadas, 15 correspondieron al sector privado $(16,1 \%)$.

Dentro de los fallecidos que pertenecían al sistema privado, para el año 2001 los hombres alcanzaron al 97,5\% y las mujeres a 2,5\%, el 2002 correspondieron $100 \%$ a hombres y el 2003 los hombres registraron $98,9 \%$ y las mujeres $1,1 \%$. 
Tabla 10. Número de casos de infección por VIH/SIDA en comunas, según nivel de logro en el índice de desarrollo humano. Defunciones no notificadas. Ambos sexos. Chile, 1997-2003

\begin{tabular}{|c|c|c|c|c|c|c|c|}
\hline IDH $* *$ & Comunas & Casos & Orden IDH & IDH* & Comunas & Casos & Orden IDH \\
\hline 1 & Vitacura & 4 & 1 & 1 & Valparaíso & 6 & 80 \\
\hline 1 & Providencia & 8 & 2 & 1 & Los Ángeles & 1 & 81 \\
\hline 1 & Las Condes & 7 & 3 & 1 & Puerto Montt & 1 & 82 \\
\hline 1 & Lo Barnechea & 1 & 4 & 1 & El Bosque & 6 & 87 \\
\hline 1 & $\underset{\sim}{\text { La Reina }}$ & 1 & 5 & 1 & Pedro Aguirre Cerda & 3 & 88 \\
\hline 1 & Ñuñoa & 6 & 6 & 1 & La Ligua & 1 & 90 \\
\hline 1 & Santiago & 21 & 11 & 1 & San Antonio & 3 & 91 \\
\hline 1 & Concepción & 3 & 12 & 1 & Padre Hurtado & 1 & 92 \\
\hline 1 & La Florida & 24 & 14 & 1 & Curacaví & 1 & 99 \\
\hline 1 & Viña del Mar & 13 & 16 & 1 & Colina & 3 & 104 \\
\hline 1 & Temuco & 3 & 17 & 1 & Quilicura & 5 & 105 \\
\hline 1 & La Cisterna & 3 & 19 & 1 & San Ramón & 3 & 107 \\
\hline 1 & Machalí & 3 & 20 & 1 & Casablanca & 1 & 112 \\
\hline 1 & Macul & 6 & 22 & 1 & Renca & 17 & 114 \\
\hline 1 & La Serena & 3 & 23 & 1 & Los Vilos & 1 & 115 \\
\hline 1 & San Miguel & 3 & 24 & 1 & La Pintana & 5 & 120 \\
\hline 1 & Maipú & 17 & 25 & 1 & Osorno & 3 & 121 \\
\hline 1 & Iquique & 9 & 28 & 1 & Nogales & 1 & 123 \\
\hline 1 & San José de Maipo & 1 & 29 & 1 & Ovalle & 3 & 125 \\
\hline 1 & Villa Alemana & 2 & 30 & 1 & Melipilla & 3 & 126 \\
\hline 1 & Antofagasta & 1 & 31 & 2 & Cerro Navia & 8 & 135 \\
\hline 1 & Cerrillos & 4 & 34 & 2 & Linares & 1 & 137 \\
\hline 1 & Punta Arenas & 1 & 35 & 2 & Rengo & 1 & 138 \\
\hline 1 & Lo Prado & 2 & 37 & 2 & Penco & 1 & 152 \\
\hline 1 & Puente Alto & 13 & 39 & 2 & Isla de Maipo & 2 & 156 \\
\hline 1 & Diego de Almagro & 1 & 40 & 2 & Lo Espejo & 6 & 161 \\
\hline 1 & Los Andes & 2 & 41 & 2 & Doñihue & 2 & 163 \\
\hline 1 & Rancagua & 12 & 42 & 2 & Lampa & 1 & 173 \\
\hline 1 & Peñalolén & 16 & 43 & 2 & Requínoa & 1 & 186 \\
\hline 1 & Calama & 1 & 46 & 2 & El Monte & 3 & 188 \\
\hline 1 & Arica & 2 & 47 & 2 & Pichidegua & 1 & 200 \\
\hline 1 & San Joaquín & 1 & 49 & 2 & Santa Cruz & 2 & 207 \\
\hline 1 & Estación Central & 6 & 50 & 2 & San Pedro de Atacama & 1 & 209 \\
\hline 1 & Recoleta & 10 & 53 & 2 & Cauquenes & 1 & 213 \\
\hline 1 & Valdivia & 1 & 54 & 2 & Tomé & 2 & 217 \\
\hline 1 & Paine & 1 & 55 & 2 & Arauco & 1 & 220 \\
\hline 1 & Coquimbo & 3 & 56 & 2 & Laja & 1 & 221 \\
\hline 1 & Talca & 3 & 58 & 2 & Calbuco & 2 & 222 \\
\hline 1 & Til-Til & 1 & 59 & 2 & Romeral & 1 & 223 \\
\hline 1 & San Bernardo & 6 & 60 & 2 & Lota & 4 & 230 \\
\hline 1 & Copiapó & 2 & 63 & 3 & Molina & 3 & 234 \\
\hline 1 & Peñaflor & 2 & 64 & 3 & Curanilahue & 1 & 238 \\
\hline 1 & Limache & 2 & 66 & 3 & Petorca & 1 & 241 \\
\hline 1 & Conchalí & 11 & 67 & 3 & Victoria & 1 & 255 \\
\hline 1 & Quinta Normal & 8 & 68 & 3 & San Clemente & 1 & 280 \\
\hline 1 & La Granja & 3 & 69 & 3 & Lebu & 1 & 289 \\
\hline 1 & Independencia & 10 & 70 & 3 & Santa Barbara & 1 & 293 \\
\hline 1 & Talcahuano & 5 & 71 & & Chiguayante & 1 & $*$ \\
\hline 1 & Tocopilla & 2 & 72 & & Concón & 2 & $*$ \\
\hline 1 & Quillota & 1 & 74 & & San Pedro de la Paz & 2 & $*$ \\
\hline 1 & Pudahuel & 9 & 76 & & Total & 404 & 100 \\
\hline
\end{tabular}

* comunas nuevas las cuales no alcanzaron a ser incluidas en el "estudio de desarrollo humano en las comunas de Chile. MIDEPLAN/PNUD, 2000".

** Clasificación de IDH estratificado según ranking: $1=1-133 \quad 2=134-233 \quad 3$ =234-333 


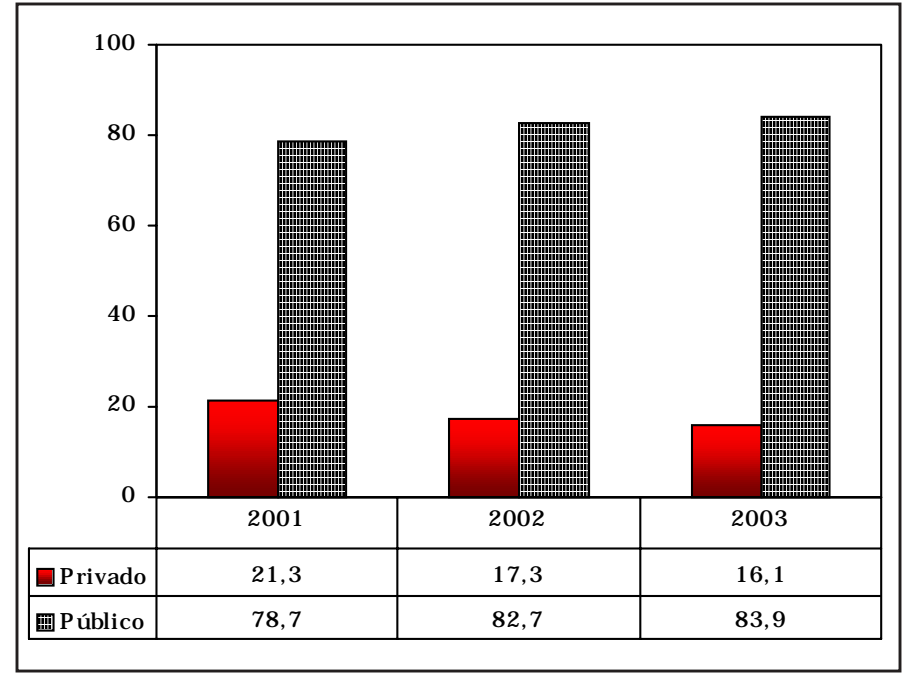

Gráfico 8. Distribución de fallecidos por SIDA no notificados, según afiliación a sistema público o privado de salud. Ambos sexos. Chile, 2001-2003.
El gráfico 8 muestra la distribución porcentual de los fallecidos por SIDA no notificados y su pertenencia según sea sistema de salud público o privado. El promedio para los años 2001-2003 de afiliados al sistema privado fue de $18,2 \%$ y al sistema público $81,8 \%$. Entre los años 2001 y 2002 se produjo un incremento de 5,9\% en el sector privado; sin embargo, éste disminuyó en los años 2002-2003 en 16,7\%. Si bien se observó un aumento de las defunciones no notificadas en el sistema público y disminución en el sistema privado, no podemos determinar una tendencia dado la escasa cantidad de años analizados.

No se dispone de igual análisis para las defunciones Notificadas.

Correspondencia a:

Edith Ortiz

eortiz@minsal.cl 